\title{
THE ELECTROENCEPHALOGRAM IN ELECTRICALLY INDUCED CONVULSIONS IN RABBITS
}

\author{
BY
}

\author{
H. LÖWENBACH and R. S. LYMAN
}

From the Henry Phipps Psychiatric Clinic, Johns Hopkins Hospital, Baltimore, Md., U.S.A.

\section{(Received 28Th August, 1940)}

THAT it is easily possible to produce generalized convulsions of short duration in animals with alternating current of high voltage, applied for a short time directly to the intact head, has been known for several years. In 1938 Krasnogorsky reported the influence of convulsions produced by such means on the behaviour of dogs. Spiegel, in 1936, described in detail a method by which one can determine the "convulsive threshold" of an animal. In experiments designed to test the anti-convulsive effect of different drugs, Merritt and Putnam (1938) produced convulsions by using an interrupted rectangular direct current given 80 times per second. The many studies which were undertaken under the auspices of the " Conference on Electric Shock," and were published between 1927 and 1930, are for our special purpose without direct interest; these authors (especially O. R. Langworthy and W. B. Kouwenhowen) investigated the effect of much higher voltages (500-2,000 volts) upon the respiratory, circulatory, and nervous system. The position of the electrodes on the head differed for each author, but apparently played no rôle as to the type of convulsions produced. Krasnogorsky (1938) placed one electrode on the top of the head between the ears and the other under the jaw. Spiegel (1936) inserted them into the conjunctival sacs of both eyes because former experiments had shown that there the resistance was smaller and less variable. Merritt and Putnam (1938) fastened one electrode in the mouth, the other over the occiput. Cerletti and Bini undertook a series of experiments in dogs in which generalized convulsions by essentially the same method as that described by Spiegel were produced. Following these experiments they applied a similar procedure to humans in the treatment of certain psychotic states (Cerletti and Bini, 1938). Since then this method in place of the convulsive treatment with metrazol has been employed in several clinics (Kalinowsky (1939), Sogliani (1939), Müller (1940), Golla et al. (1940)).

Before adopting this therapeutic procedure ourselves it was thought advisable to study the physiological processes in these electrically induced convulsions in animals. The electroencephalograph was chosen as the best available indicator of cortical function. 


\section{Method}

Rabbits were subjected to the following operation under local anæsthesia: skin, muscle, and periosteum were removed from the top of the head over an area of 2 by $4 \mathrm{~cm}$. Six holes were drilled electrically just deep enough to insert fine screws to which the recording electrodes were attached. This was desirable for two reasons. First, it was necessary to ensure that the electrodes did not become loose during the seizures, and secondly, it allowed comparison of the results of shocks given on different days to the same animal. In some experiments a seventh electrode was similarly placed in the occipital bone as an "inactive" electrode for " unipolar" connection. An early attempt to fasten this " inactive" electrode to a place remote from the head, such as the ear or a shaven part of the body, had to be given up because of the interference from muscular action-currents during the seizures. The electrodes, either unipolar or bipolar, were connected through an amplifier system to an ink-writing recorder constructed by Mr. A. M. Grass. As six amplifier systems were at our disposal the electrocardiogram and the muscular action-currents of one fore-leg were recorded simultaneously in many experiments. Owing to disturbance produced by the alternating current, the recording was begun about a second after each shock.

The stimulating or " shock" electrodes were placed directly above the hind-angle of each eye after the hair had been shaved and a small amount of electro-jelly rubbed into the skin. Every effort was made to ensure that these electrodes were as near as possible in the same spot in the different rabbits, and in the same rabbit on subsequent days. Some of the minor differences in the results may be ascribed to slight variation in position of the electrodes. The main supply was reduced by means of a variable transformer (Variac Type 200B), and an X-ray time switch (Type Wappler-Impex) regulated the duration of the current. Under these standard conditions the voltage necessary to produce a generalized seizure lay between 25 and 35 volts, and the duration mostly $\frac{1}{10}$ second. Before each experiment the resistance to alternating current was determined approximately.

During each experiment the animal was tied to an animal-board, and the head immobilized by a Cermak's clamp. An additional bandage had to be put around the mouth in order to prevent it slipping out of the clamp during the initial stage of the convulsion. The convulsion produced had similar characteristics to those described by Spiegel (1936); a detailed description therefore seems unnecessary.

\section{Results}

Before each "shock" was given, a control electroencephalogram was taken. In most of our animals the recording electrodes lay over the area præcentralis agranularis, and the familiar response pattern of this area was recorded. When care was taken to exclude as far as possible all outside disturbances, characteristic runs of fast waves were present, sometimes with a longer, sometimes a shorter rhythm. In some of the animals these runs were either never present or they disappeared a few days after the first electroencephalogram had been taken. It was common to find in such a case that either the drill had gone too deep and had injured the surface of the brain or an infection had developed.

The "shock" was then given. With the click of the switch, the animal jumped in its bandage, and then stretched out into an opisthotonus, tail erect, as far as the ties would allow it. Not infrequently fæces and urine were ejected. Within 6-10 seconds this state passed, without marked interruption, into a series of clonic contractions, the number of which varied in the same animal, and from animal to animal. The pupils were wide, the corneal reflex was 
absent. When the clonic phase was over, the animal lay with all muscles relaxed and the tail down. Usually slow conjugate oscillations of the eyes from side to side could be observed for a varying time after the clonic phase. During the first tonic state, respiration ceased; the clonic phase was accompanied by inspirations and expirations; later the respiratory rate became rapid but gradually returned to its original rate.

In the electroencephalogram the tonic phase made itself evident by a continuous sequence of large spikes with a frequency of 3-10 per second. Towards the end of this phase the spikes were spaced wider and wider apart and became more triangular in shape (Fig. 1). The clonic phase was marked by a single or double spike, fast in the upward and slow in the downward phase on the

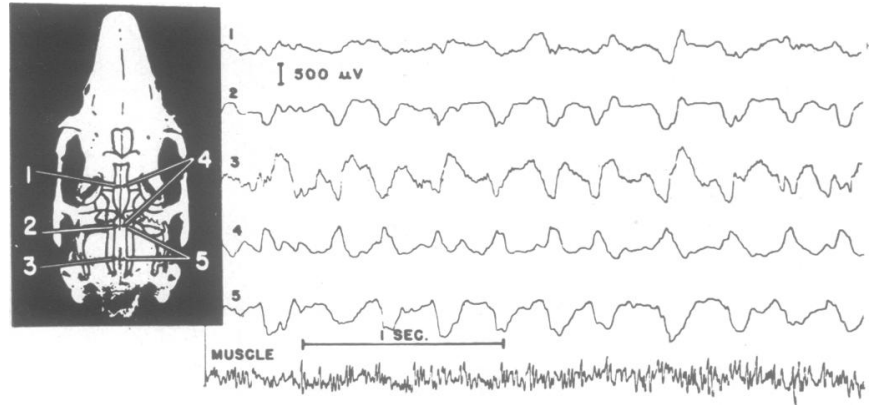

Fig. 1.-30 volts, given for $\frac{1}{10}$ second. 10 seconds after shock. " Tonic" phase.

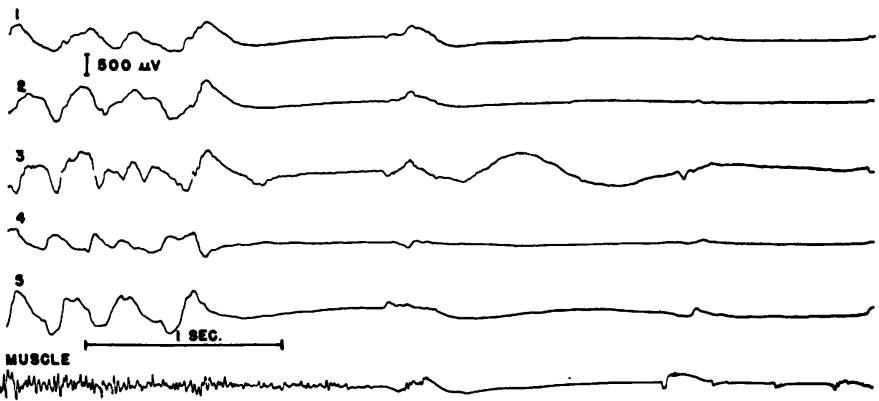

Fig. 2.-The same animal 19 seconds after the shock. The "tonic" phase passes into the "clonic" one.

record. All other fast oscillations had disappeared (Fig. 2). These eruptions were followed by a period in which either all electrical activity ceased and the oscillograph wrote an almost straight line, or slow swings, of low voltage, were present. This latter period lasted from one to several seconds (Fig. 3). From the resulting straight line, slow swings (one per second and less) emerged and became gradually bigger and faster until finally the whole pattern consisted of large 2-3 per second waves (Fig. 4). In some experiments the original pattern was resumed within 1 minute; in others the period of slow waves continued for several minutes (Fig. 5). At this stage every disturbance had to be avoided, even more so than during the control run; otherwise, the slightest noise, e.g. the sliding of the recording paper down to the floor or a whisper between 
the experimenters, might instantaneously destroy the fast runs just reappearing and keep the pattern dominated by slow waves only.

During the tonic phase the records from the muscles showed a continuous discharge of large and fast-action currents. Towards the end of the tonic phase,

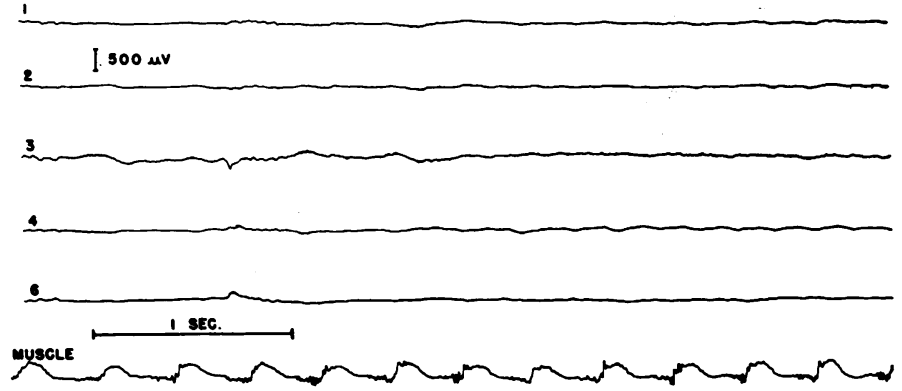

Fig. 3.-Direct continuation of Fig. 2. Notice the clonic discharges in the record from the muscle, while the monopolar and bipolar records from six different points of the motor cortex are almost flat.

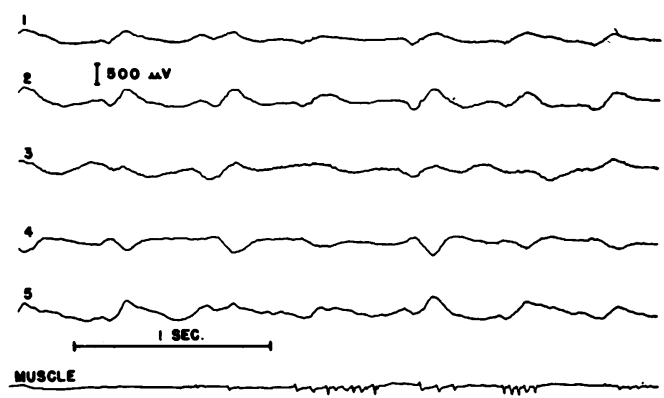

Fig. 4. -57 seconds after the shock. Beginning recovery of the cerebral electro-activity.

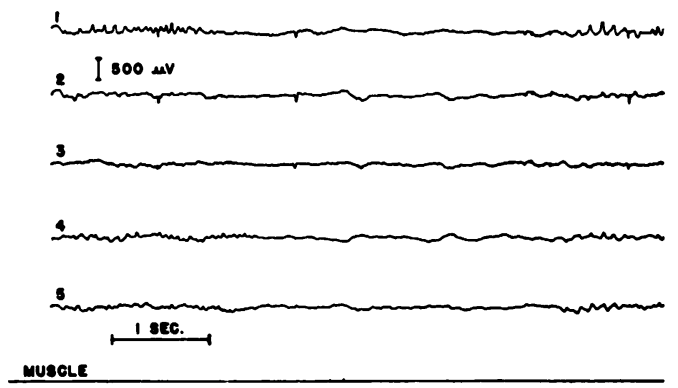

Fig. 5.-First reappearance of the normal activity of the area præcentralis agranularis; 185 seconds after the shock.

these action currents gradually faded out and were almost entirely absent before the first clonic jerk. Each of the cerebral discharges was accompanied by a simultaneous muscular one. But when the brain had ceased to show electrical activity (the " silent period" just after the end of the clonic phase), we observed many times a series of muscular contractions of clonic character which was not accompanied by any cerebral equivalent. This was observed with both 
bipolar and monopolar connections. Finally, half an hour after the shock no signs of disturbed electrical activity were to be found in the records. Nor were there any symptoms of abnormal behaviour when the animal was let free from the table. When the animal was freed prior to the full return of the " normal" pattern, it would either stumble from one side to the other, even fall, or it would sit motionless and not move even when pushed.

Occasionally the stimulus, intentionally or accidentally, did not provoke a fully developed convulsion. The clinical picture in such an instance consisted only of one lightning-like contraction of all muscles; thereafter the animal lay quietly but with a more frequent but less deep respiratory rhythm. The electroencephalogram showed slow waves with large amplitude of the same sort as described for the post-convulsive phase in any other type of attack. We never observed a " silent period" under these conditions. It is not possible, however, to exclude with certainty its presence during the short period before the ink-writers were turned on. The fast runs reappeared in the same gradual fashion as in the experiments with long-lasting seizures, but the interval with slow and disorganized waves was much shorter.

\section{Discussion}

When we compare our findings with the electroencephalograms during convulsions produced by metrazol, as first described by Fischer and Löwenbach (1934) in animals and recently again by Goodwin, Kerr, and Lawson (1940), by Cook and Walter (1938), and by Rubin and Wall (1939) in man, we note that the records resemble each other closely. The description of the attacks could well be that of an electrically produced seizure. The brain, once induced to discharge its energy explosively, seems to act in the same way regardless of how the explosion is set off. It even seems that the type of reaction is the same for rabbit and cat as it is for man. After a continuous display of maximal activity (successive spikes), the tiring cells appear to fire seriatim at longer and longer intervals (clonic bursts), until final exhaustion prevents all further activity (silent period). Restitution is indicated by the gradual reappearance of at first slow and later faster waves with the final return to the normal activity.

There is, however, one basic difference between the two pictures. When metrazol has been injected, there is a period, sometimes extending up to 10 seconds, during which the amplitude of the curves becomes gradually larger, and the frequency of these mounting waves becomes faster before the salvo of spikes breaks loose. When alternating current is used the peak is reached instantaneously. No latent period is required for the concentration of the convulsive agent to attain its effective level. In the treatment of patients, this point is of great practical importance. It is during this latent period that the patient experiences anxiety and the anticipation of impending danger which so many patients afterwards recall with distress. Moreover, these sensations are not blotted out by the amnesia which comes to cover the time of the convulsion proper. With the electrical method the absence of these sensations, as reported by all authors who have made use of it so far, is explained 
by the suddenness of the onset of the cerebral disturbance at the moment the switch closes the circuit.

Cook and Walter, on the one hand, and Rubin and Wall, on the other, disagree on the question whether clonic movements occur without a cerebral electrical equivalent. The former saw clonic contractions of the muscles when the electrical activity of the brain had already ceased. Rubin and Wall, who recorded simultaneously the muscular-action currents, were unable to substantiate this. They believe that the results of Cook and Walter are explained by the fact that the latter used bipolar connections only, and that the straight line in the electroencephalographic record during the clonic phase is a consequence of a strictly synchronous activity of the cortex. We have seen too often the action currents associated with clonic muscular contractions continuing into the "silent period" of cerebral action potentials with monopolar as well as bipolar connections to regard the "silent period" as a fault of the method. We feel sure that at least some of the muscular contractions are not of cortical origin. One might further expect that the huge spikes occurring during the tonic phase would have to be produced by " synchronous " activity in the central nervous system, and that bipolar connections, therefore, would show isoelectrical waves. We think that the tonic state originates entirely in the cerebrum, and is the muscular expression of rapid discharges of cortical centres. The clonic contractions, however, may arise from activity of the nervous system at lower levels, even from the segmental motor neuronescomparable, perhaps, to the muscular twitches after application of strychnineat a time when the higher parts of the brain are already fully exhausted. This view is in accord with the findings of Samaya (1903), who reported that clonic convulsions may be induced in rabbits and other animals by electrical stimulation of the medulla oblongata. In man, Jasper and Andrews (1936), recording changes in cerebral potentials and muscular action currents at the same time, saw occasionally clonic movements which appeared quite independently from the seizure phenomena on encephalographic records.

In none of our experiments did we find changes of the pattern of changes in cerebral electrical potentials which lasted longer than the clinical picture would lead us to expect. Animals which were given 16 convulsions within 22 days showed much the same " resting" pattern each time before the current was applied. Anatomical studies on the same material have been begun and will be recorded after they have been completed.

\section{Summary}

Rabbits were subjected to convulsions induced by 60 -cycle alternating current (25-35 volts, usually for $-\frac{1}{10}$ second). Cerebral action potential changes were recorded from electrodes fixed in the bone of the skull, with all overlying muscle removed.

In the tonic phase of the convulsions, which came immediately after administering the current, the records showed large spikes, at first in a continuous run (3-10 per second). Then these became spaced farther apart, $2 \mathrm{~A}$ 
until one or more spikes appeared only at intervals, synchronous with the jerks of the clonic phase. Then all significant oscillations usually stopped (" silent period" of cortical activity). However, clonic contractions of the muscles, simultaneously recorded electrographically, not infrequently extended into this "silent period." The changes in cerebral action potential then returned after seconds or a few minutes and went through slowly swinging deflections to arrive gradually at the usual pattern in the course of some minutes to about half an hour.

These phenomena are interpreted as indicating immediate, almost continuous, maximal discharges of cortical cells, then seriatim firing at longer intervals as the cells tire, followed by cessation of all demonstrable activity with exhaustion. The muscular action currents of the clonic twitches may continue into the "silent period" of cerebral exhaustion, so that at least some of the later muscular contractions may arise from discharges originating at lower levels of the nervous system than the cortex.

Preliminary runs were taken before giving each convulsion and occasional records were obtained after the series of 16 convulsions had been completed. During the period of observation no appreciable change could be noted in the records of changes in cerebral action potentials obtained before and after the convulsion.

The main difference in convulsions induced electrically and those produced by metrazol consists in the absence of a latent period and the wider range of duration and severity which can be obtained in the former. Convulsions come immediately after closing the electrical circuit, and the effect may be made much shorter and milder than is possible in a metrazol convulsion.

\section{REFFERENCES}

Cerletti, U., and Bini, L. (1938). Boll. Acad. med., Roma, 136.

Cook, L. C., and Walter, W. G. (1938). J. Neurol. Psychiat., 1, 180.

Fischer, M. H., and Löwenbach, H. (1934). Arch. exp. Path. Pharmak., 174, 502.

Golla, F., Walter, W. G., and Fleming, G. W. (1940). Proc. roy. Soc. Med., 33, 261.

Goodwin, J. E., Kerr, W. K., and Lawson, F. L. (1940). Amer. J. Psychiat., 96, 1389.

Jasper, H. H., and Andrews, H. L. (1936). Quant. Biol., 4, 320.

Kalinowsky, L. (1939). Lancet, 1232.

Krasnogorsky, N. I. (1938). Proc. 15th int. Physiol. Congr., 239.

Merritt, H. H., and Putnam, T. J. (1938). Arch. Neurol. Psychiat., Chicago, 39, 1003.

Müller, M. (1940). Schweiz. med. Wschr., 70, 323.

Rubin, M. A., and Wall, C. (1939). J. Neurol. Psychiat., 2, 107.

Samaya, N. (1903). Trav. Lab. Physiol., Geneve, 4, 49. (Quoted from Blume, W. (1926). Arch. exp. Path. Pharmak., 116, 234.)

Sogliani, G. (1939). Rass. Studi psichiat., 28, 652.

Spiegel, E. A. (1936). J. Lab. clin. Med., 22, 1274. 